\title{
新型冠状病毒肺炎疫情下武汉及周边地区何时 复工？数据驱动的网络模型分析
}

\author{
王霞 ${ }^{1}$ ，唐三一 ${ }^{1}$, 陈勇 ${ }^{2}$, 冯晓梅 ${ }^{3}$, 肖燕妮 ${ }^{4 *}$, 徐宗本 ${ }^{5}$ \\ 1. 陕西师范大学数学与信息科学学院, 西安 710119 ; \\ 2. 中国人民解放军疾病预防控制中心, 北京 100071 ; \\ 3. 运城学院数学与信息技术学院, 运城 044000 ; \\ 4. 西安交通大学数学与统计学院, 数学与生命科学交叉中心, 西安 710049 ; \\ 5. 西安交通大学数学与统计学院, 大数据算法与分析技术国家工程实验室, 西安 710049 \\ E-mail: xiawang@snnu.edu.cn, sytang@snnu.edu.cn, chenyonger@126.com, xiaomei_0529@126.com, \\ yxiao@mail.xjtu.edu.cn,zbxu@mail.xjtu.edu.cn
}

收稿日期: 2020-02-14；接受日期: 2020-02-17；网络出版日期: 2020-02-20；＊通信作者 国家自然科学基金 (批准号: 11631012, 61772017 和 11601301) 资助项目

\begin{abstract}
摘要＼cjkstart基于全国和湖北省新型冠状病毒肺炎 (COVID-19) 疫情报告数据以及百度人口迁徙与分布大 数据, 本文构建武汉及周边 15 个疫情严重城市的 COVID-19 传播复杂网络模型, 重点分析武汉及周 边地区复工的可能时间节点和复工对二次暴发风险的影响. 首先基于各个城市的累计病例数估计 1 月 23 日武汉的累计病例数, 得到不同时期湖北省 16 个主要城市控制再生数的估计值, 揭示了早期的传 播风险较大和目前的传播风险小 (控制再生数的值小于 1). 本文基于 2019 年同期的流动网络结构和 流动量模拟整个网络模型, 给出 2020 年 2 月 17 日、2月 24 日和 3 月 2 日的复工对各个城市疫情的 影响. 主要结论显示, 在较强的防控措施和自我防护下, 2020 年 3 月 2 日复工将在一段时间内不会引 起疫情的二次暴发.
\end{abstract}

关键词 新型冠状病毒肺炎 网络模型 控制再生数 疫情预测

MSC (2010) 主题分类 $35 \mathrm{~A} 34,65 \mathrm{~N} 21$

\section{1 引言}

在 2020 年春节前暴发的 COVID-19 疫情 (参见文献 [1,2]), 由武汉首发并快速向全国蔓延, 人 民群众的正常生活和社会经济遭受重大影响, 生命健康受到极大的威胁. 现如今, 举国上下众志成城, 亿万军民勠力同心, 全面打响了夺取疫情防控的阻击战. 这一过程中, 数学模型在早期 COVID-19 疫 情的预测、预警和风险分析中具有非常重要的作用. 具体体现在, 利用少量和实时更新的数据, 构建

英文引用格式: Wang X, Tang S Y, Chen Y, et al. When will be the resumption of work in Wuhan and its surrounding areas during COVID-19 epidemic? A data-driven network modeling analysis (in Chinese). Sci Sin Math, 2020, 50: 969-978, doi: 10.1360/SSM-2020-0037 
符合 COVID-19 传播和我国特有的防控策略的数学模型, 发展包括数据处理、最小二乘法和 MCMC (Markov chain Monte Carlo) 方法等统计计算方法, 确定模型未知参数, 最终实现: (1) 确定传播风险指 标 (基本再生数)、达峰时间、峰值和最终感染规模等在内的疫情技术指标; (2) 评估包括封城、密切跟 踪隔离、检测和检出等重大防控策略的有效性和时效性 (参见文献 [3-5]). 上述目标的实现能为疾病 预防控制和决策部门提供重要的决策依据, 服务于国家重大突发性传染性疾病防控, 具有明确的理论 和现实意义.

由于 COVID-19 疫情在 2020 年春节前夕暴发, 武汉于 2020 年 1 月 23 日凌晨才开始实施封城策 略, 而全国其他地区限制人员流动的策略自 1 月 23 日以后才逐步实行. 早期湖北省内的孝感市、黄 冈市、荆州市和咸宁市等众多地区的疫情都以输入病例为主, 省外如北京市和陕西省的早期病例绝大 比例也是输入病例. 由此可见, 在人们真正了解疫情之前, 出现发热症状或潜伏期人员已经从武汉流 出. 通过近 20 天各地市的严格防控, 全国的新报告病例数已呈现下降趋势. 春节后的复工和复学也提 上日程. 传染病预防控制与决策部门高度关注的核心问题是: 武汉及周边疫情严重的地区何时复工及 复工的最佳时间是什么? 早复工对疫情特别是武汉及周边主要城市疫情的影响是什么?

为了回答上述问题、为决策部门提供何时复工等重要决策依据, 我们收集了湖北省卫生健康委员 会 (简称卫健委) 和国家卫健委官网上报道的全国以及湖北各地市的疫情数据, 收集了百度迁徙网站 (http://qianxi.baidu.com) 人口流动数据, 以及春运期间武汉人口迁入和迁出趋势和流入到湖北其他地 市的人口分布情况. 基于文献 [4-6] 中的 COVID-19 疫情传播与控制模型框架, 发展以武汉为中心的 复杂网络模型, 通过统计计算与参数估计确定网络模型未知参数, 分析武汉及周边 15 个疫情严重地 区复工的最佳时间, 评估早复工对这些地区疫情发展特别是二次暴发风险的影响. 主要结论显示 3 月 2 日复工再加上较强的防控措施将不会引起疫情的二次暴发, 而早于这个时间二次暴发的风险较大.

\section{2 数据、模型与参数估计}

主要数据 自 COVID-19 疫情暴发以来, 湖北省卫健委和国家卫健委通报了疫情数据, 包括每 天新增病例数、累计报告病例数、累计治愈病例数、累计死亡病例数和疑似病例数等. 我们主要收集 了全国以及湖北各地市的数据 ${ }^{[7,8]}$. 此外, 为了考虑武汉的输出病例对湖北其他地市疫情的影响, 我 们从百度迁徙网站收集了人口流动数据, 主要包括春运期间武汉迁入和迁出趋势以及迁入到湖北其 他地市的人口分布情况 (见图 1 和 2). 为了确定疾病暴发初期易感者的人数, 我们从百度百科网站 (https://baike.baidu.com) 收集了湖北各地市的人口数量 (见表 1 ).

动力学模型 基于疾病的临床进展、流行病学状况以及政府采取的控制措施的情况, 我们建立了 一个 SEIR 仓室模型. 考虑将自然传播过程的人群分为易感者类 $(S)$ 、潜伏者类 $(E)$ 、感染者类 (有症

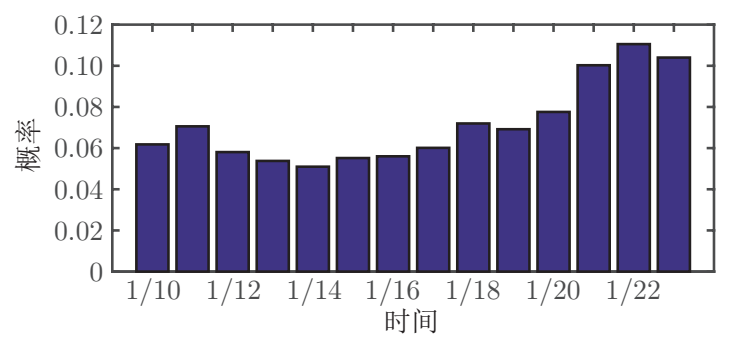

(a)

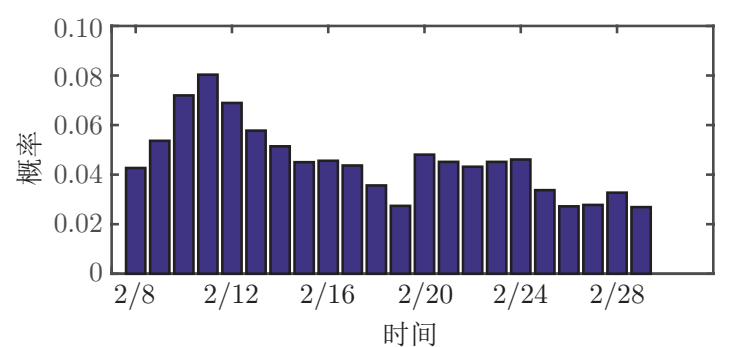

(b)

图 1 (网络版彩图) (a) 2020 年春节前武汉的人口迁出时间分布; (b) 2019 年春节后武汉的人口迁入时间分布 

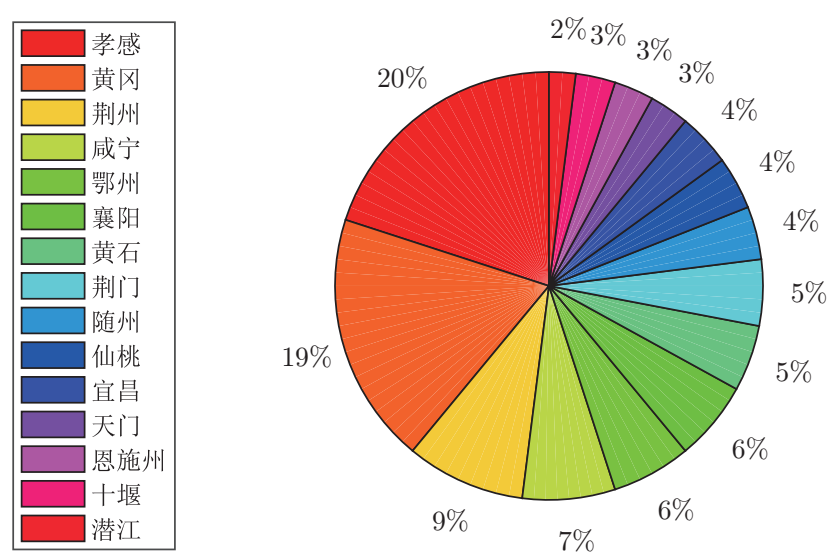

图 2 (网络版彩图) 2020 年 1 月 10 日至 1 月 23 日武汉迁出人口目的地平均分布

表 1 各城市总人口数

\begin{tabular}{cccccccc}
\hline 武汉 & 孝感 & 黄冈 & 荆州 & 咸宁 & 鄂州 & 襄阳 & 黄石 \\
\hline 11081000 & 5300000 & 7500000 & 6410000 & 2535100 & 1076900 & 6050000 & 2689300 \\
$S_{1}(0)$ & $S_{2}(0)$ & $S_{3}(0)$ & $S_{4}(0)$ & $S_{5}(0)$ & $S_{6}(0)$ & $S_{7}(0)$ & $S_{8}(0)$ \\
\hline \hline 荆门 & 随州 & 仙桃 & 宜昌 & 天门 & 恩施州 & 十堰 & 潜江 \\
\hline 3000000 & 2216700 & 1563500 & 4169200 & 1609200 & 4026100 & 3406000 & 962000 \\
$S_{9}(0)$ & $S_{10}(0)$ & $S_{11}(0)$ & $S_{12}(0)$ & $S_{13}(0)$ & $S_{14}(0)$ & $S_{15}(0)$ & $S_{16}(0)$ \\
\hline
\end{tabular}

状) $(I)$ 、感染者类 (无症状) $(A)$ 、住院者类 $(H)$ 和恢复者类 $(R)$. 由于密切跟踪隔离措施的实施, 与 感染者密切接触的人群分为隔离的易感者类 $\left(S_{q}\right)$ 和潜伏者类 $\left(E_{q}\right)$. 通过感染者密切接触追踪, 假设 $q$ 比率的接触者被隔离, 其中被隔离的个体若被感染, 则该个体隔离在 $E_{q}$ 仓室, 否则隔离在 $S_{q}$ 仓室. 若比率为 $1-q$ 的接触者在追踪中被遗漏, 一旦被有效感染则移动到 $E$ 仓室, 否则仍然留在仓室 $S$ 中. 设每次接触时传播概率为 $\beta$, 接触数为 $c$. 被隔离的个体中, 如果被感染 (或未受感染), 则以 $\beta c q$ (或 $(1-\beta) c q$ ) 的速率移动到仓室 $E_{q}$ (或 $S_{q}$ ). 若未被隔离且被感染, 则以 $\beta c(1-q)$ 的速率转移到仓室 $E$. 感染者个体被确诊的速率为 $\delta_{I}$, 并以 $\gamma_{H}$ 的速率恢复到仓室 $R$. 具体模型如下所示:

$$
\left\{\begin{array}{l}
\frac{d S}{d t}=-(\beta c+c q(1-\beta)) S(I+\theta A+\nu E)+\lambda S_{q}, \\
\frac{d E}{d t}=\beta c(1-q) S(I+\theta A+\nu E)-\sigma E, \\
\frac{d I}{d t}=\sigma \rho E-\left(\delta_{I}+\alpha+\gamma_{I}\right) I, \\
\frac{d A}{d t}=\sigma(1-\rho) E-\gamma_{A} A, \\
\frac{d S_{q}}{d t}=(1-\beta) c q S(I+\theta A+\nu E)-\lambda S_{q}, \\
\frac{d E_{q}}{d t}=\beta c q S(I+\theta A+\nu E)-\delta_{q} E_{q}, \\
\frac{d H}{d t}=\delta_{I} I+\delta_{q} E_{q}-\left(\alpha+\gamma_{H}\right) H, \\
\frac{d R}{d t}=\gamma_{I} I+\gamma_{A} A+\gamma_{H} H,
\end{array}\right.
$$

参数定义见表 2 . 为了考虑武汉感染者的流出对湖北其他地市疫情的影响, 我们将上述 SEIR 模型进 
表 2 参数定义和估计值

\begin{tabular}{|c|c|c|c|}
\hline 参数 & 定义 & 参数值 & 来源 \\
\hline$c$ & 接触率 & 13.0046 & 参数估计 \\
\hline$\beta$ & 每次接触传播的概率 & $2.0389 \times 10^{-9}$ & 参数估计 \\
\hline$q$ & 隔离率 & $1.8877 \times 10^{-7}$ & 参数估计 \\
\hline$\sigma$ & 潜伏者到感染者的转移率 & $1 / 5$ & 文献 [9] \\
\hline$\lambda$ & 隔离的未受感染接触者释放回社区的速率 & $1 / 14$ & 文献 $[7,10]$ \\
\hline$\rho$ & 感染者有症状的概率 & 0.6834 & 参数估计 \\
\hline$\delta_{I}$ & 有症状的感染者被隔离的速率 & 0.1328 & 参数估计 \\
\hline$\delta_{q}$ & 隔离的潜伏者变为隔离的感染者的速率 & 0.1259 & 参数估计 \\
\hline$\gamma_{I}$ & 有症状的感染者的恢复率 & 0.1029 & 参数估计 \\
\hline$\gamma_{A}$ & 无症状感染者的恢复率 & 0.2978 & 参数估计 \\
\hline$\gamma_{H}$ & 隔离的感染者的恢复率 & 0.1024 & 参数估计 \\
\hline$\alpha$ & 因病死亡率 & 0.0009 & 参数估计 \\
\hline$\theta$ & 无症状感染者接触率调节因子 & 1.6003 & 参数估计 \\
\hline$\nu$ & 潜伏者接触率调节因子 & 1.5008 & 参数估计 \\
\hline 初值 & 定义 & 参数值 & 来源 \\
\hline$S_{1}(0)$ & 易感者的初值 & 11081000 & 文献 [9] \\
\hline$E_{1}(0)$ & 潜伏者的初值 & 600.0110 & 参数估计 \\
\hline$I_{1}(0)$ & 有症状感染者的初值 & 409.9978 & 参数估计 \\
\hline$A_{1}(0)$ & 无症状感染者的初值 & 30.0278 & 参数估计 \\
\hline$S_{q 1}(0)$ & 隔离的易感者的初值 & 739 & 数据 \\
\hline$E_{q 1}(0)$ & 隔离的潜伏者的初值 & 20 & 参数估计 \\
\hline$H_{1}(0)$ & 隔离的感染者的初值 & 41 & 数据 \\
\hline$R_{1}(0)$ & 恢复者的初值 & 2 & 数据 \\
\hline
\end{tabular}

一步拓展为如下网络模型:

$$
\left\{\begin{array}{l}
\frac{d S_{1}}{d t}=-(\beta c+c q(1-\beta)) S_{1}\left(I_{1}+\theta A_{1}+\nu E_{1}\right)+\lambda S_{q 1}-\sum_{i=2}^{n} m_{i}^{S}(t) \\
\frac{d E_{1}}{d t}=\beta c(1-q) S_{1}\left(I_{1}+\theta A_{1}+\nu E_{1}\right)-\sigma E_{1}-\sum_{i=2}^{n} m_{i}^{E}(t) \\
\frac{d I_{1}}{d t}=\sigma \rho E_{1}-\left(\delta_{I}+\alpha+\gamma_{I}\right) I_{1}-\sum_{i=2}^{n} m_{i}^{I}(t) \\
\frac{d A_{1}}{d t}=\sigma(1-\rho) E_{1}-\gamma_{A} A_{1}-\sum_{i=2}^{n} m_{i}^{A}(t) \\
\frac{d S_{q 1}}{d t}=(1-\beta) c q S_{1}\left(I_{1}+\theta A_{1}+\nu E_{1}\right)-\lambda S_{q 1} \\
\frac{d E_{q 1}}{d t}=\beta c q S_{1}\left(I_{1}+\theta A_{1}+\nu E_{1}\right)-\delta_{q} E_{q 1} \\
\frac{d H_{1}}{d t}=\delta_{I} I_{1}+\delta_{q} E_{q 1}-\left(\alpha+\gamma_{H}\right) H_{1} \\
\frac{d R_{1}}{d t}=\gamma_{I} I_{1}+\gamma_{A} A_{1}+\gamma_{H} H_{1}
\end{array}\right.
$$




$$
\left\{\begin{array}{l}
\frac{d S_{i}}{d t}=-(\beta c+c q(1-\beta)) S_{i}\left(I_{i}+\theta A_{i}+\nu E_{i}\right)+\lambda S_{q i}+m_{i}^{S}(t) \\
\frac{d E_{i}}{d t}=\beta c(1-q) S_{i}\left(I_{i}+\theta A_{i}+\nu E_{i}\right)-\sigma E_{i}+m_{i}^{E}(t) \\
\frac{d I_{i}}{d t}=\sigma \rho E_{i}-\left(\delta_{I}+\alpha+\gamma_{I}\right) I_{i}+m_{i}^{I}(t) \\
\frac{d A_{i}}{d t}=\sigma(1-\rho) E_{i}-\gamma_{A} A_{i}+m_{i}^{A}(t) \\
\frac{d S_{q i}}{d t}=(1-\beta) c q S_{i}\left(I_{i}+\theta A_{i}+\nu E_{i}\right)-\lambda S_{q i} \\
\frac{d E_{q i}}{d t}=\beta c q S_{i}\left(I_{i}+\theta A_{i}+\nu E_{i}\right)-\delta_{q} E_{q i}, \\
\frac{d H_{i}}{d t}=\delta_{I} I_{i}+\delta_{q} E_{q i}-\left(\alpha+\gamma_{H}\right) H_{i} \\
\frac{d R_{i}}{d t}=\gamma_{I} I_{i}+\gamma_{A} A_{i}+\gamma_{H} H_{i}, \quad i=2,3, \ldots, n
\end{array}\right.
$$

其中下标 1 表示武汉, 下标 $i=2,3, \ldots, n$ 分别表示孝感、黄冈、荆州、咸宁、鄂州、襄阳、黄石、荆 门、随州、仙桃、宜昌、天门、恩施州、十堰、潜江, 因此, $n=16$. 人口流动由 $m_{i}^{j}(t)$ 表示, 其中 $j$ 表 示人的状态 (易感的、潜伏的、感染的有症状或者是感染的没有症状). 由于 1 月 23 日前疫情主要发 生在武汉, 湖北其他地市没有报告病例数, 因此, 1 月 23 日前我们只考虑人口由武汉迁入到其他城市, 此时 $m_{i}^{j}(t)$ 表示人口由武汉流入第 $i$ 个城市, 并且取正值. 由于武汉在 1 月 23 日 “封城”, 因而, 假设 1 月 23 日后没有人口流动, 此时 $m_{i}^{j}(t)=0$. 考虑复工复产时人口流动重新开始, 并且春节前从武汉流 出的人返回工作岗位或学校是从各地市重新流回武汉, 此时 $m_{i}^{j}(t)$ 表示从第 $i$ 个城市流入武汉, 取负 值. 这里假设春节前大约有 500 万人流出武汉, 其中 $70 \%$ 流入到湖北其他地市, 由此再根据百度迁徙 网站中的人口流动趋势和分布来计算分段函数矩阵 $M(t)=\left\{m_{i}^{j}(t)\right\}$ 的值, 这里的矩阵 $M(t)$ 表示人口 流动的邻接矩阵 ${ }^{[11]}$. 这里分段函数共分三段, 即 1 月 10 日起至 1 月 23 日由 2020 年武汉迁出趋势 (图 1(a)) 计算, 复工后由 2019 年同阶段的武汉迁入趋势计算 (图 1(b)), 中间阶段假设没有流动, 则取 值为 0 .

参数估计 根据模型 (2.1), 利用再生矩阵的方法, 我们可以计算控制措施实施过程中的控制再生 数 ${ }^{[12]}$, 具体如下:

$$
R_{c}=\left[\frac{\beta c \rho(1-q)}{\delta_{I}+\alpha+\gamma_{I}}+\frac{\beta c(1-\rho)(1-q) \theta}{\gamma_{A}}+\frac{\beta c \nu(1-q)}{\sigma}\right] S(0)
$$

其中 $S(0)$ 表示易感者的初值. 考虑到前期人们对这种新型病毒的认识不足, 再加上医疗资源紧张, 导 致前期武汉的报告病例数相对较低, 而其他城市疫情暴发比武汉晚, 报告病例数据相比武汉较为准确, 因此, 我们基于除武汉外的其他城市的累计病例数, 采用最小二乘法来估计系统参数. 由于大部分地 区的报告病例数是从 1 月 23 日开始, 并且前三天的数据不太准确, 我们采用从 1 月 26 日至 2 月 9 日 的数据进行参数估计. 我们将数据分为三个阶段以期评估我国逐步加强的控制措施的影响, 第一阶段 (T1) 为 1 月 26 日至 1 月 30 日, 第二阶段 (T2) 为 1 月 31 日至 2 月 4 日, 第三阶段 (T3) 为 2 月 5 日至 2 月 9 日. 首先, 我们用第一阶段的数据估计模型 (2.2) 的所有参数. 为了考虑各地不断加强的控 制措施对疫情的影响, 而控制措施的影响主要体现在接触数 $c$ 和隔离率 $q$, 假设各城市间的参数 $c$ 和 $q$ 的取值不同. 固定其他参数, 分别利用这三段数据重新估计每个城市的参数 $c$ 和 $q$. 模拟的起始时间 为 1 月 10 日, 武汉的模型初值见表 2 , 其他地区易感者的初值假设为当地总人口数, 其余初值为 0 (见 表 3). 具体参数估计值和三个时间段期间的控制再生数的估计值见表 2 和 3 , 模型拟合效果见图 3 . 
表 3 各城市参数 $c$ 和 $q$ 的估计值及再生数 (其中下标 $1 、 2$ 和 3 分别表示三个时间段)

\begin{tabular}{|c|c|c|c|c|c|c|c|c|}
\hline 城市 & 武汉 & 孝感 & 黄冈 & 荆州 & 咸宁 & 鄂州 & 襄阳 & 黄石 \\
\hline$c_{1}$ & 13.40 & 15.56 & 12.36 & 12.14 & 32.65 & 70.00 & 18.63 & 34.01 \\
\hline$q_{1}$ & $1.89 \times 10^{-7}$ & $1.09 \times 10^{-9}$ & $1.05 \times 10^{-9}$ & $1.27 \times 10^{-9}$ & $7.86 \times 10^{-7}$ & $1.09 \times 10^{-9}$ & $9.73 \times 10^{-8}$ & $1.33 \times 10^{-9}$ \\
\hline$c_{2}$ & 4.48 & 13.50 & 2.00 & 33.35 & 5.01 & 66.55 & 0.10 & 30.02 \\
\hline$q_{2}$ & $4.13 \times 10^{-6}$ & $1.09 \times 10^{-6}$ & $2.46 \times 10^{-6}$ & $1.00 \times 10^{-5}$ & $4.70 \times 10^{-6}$ & $2.12 \times 10^{-9}$ & $1.00 \times 10^{-5}$ & $6.07 \times 10^{-6}$ \\
\hline$c_{3}$ & 4.48 & $1.03 \times 10^{-4}$ & 4.46 & 1.60 & 0.01 & 32.99 & 0.01 & 29.98 \\
\hline$q_{3}$ & $4.13 \times 10^{-6}$ & $1.00 \times 10^{-3}$ & $1.00 \times 10^{-3}$ & $1.00 \times 10^{-3}$ & $1.03 \times 10^{-3}$ & $1.00 \times 10^{-4}$ & $1.01 \times 10^{-5}$ & $1.08 \times 10^{-5}$ \\
\hline$R_{c 1}$ & 3.66 & 2.03 & 2.28 & 1.92 & 2.04 & 1.86 & 2.78 & 2.26 \\
\hline$R_{c 2}$ & 0.41 & 1.91 & 0.39 & 5.44 & 0.28 & 1.98 & 0.01 & 2.08 \\
\hline$R_{c 3}$ & 0.09 & $9.49 \times 10^{-6}$ & 0.77 & 0.02 & $5.00 \times 10^{-4}$ & 0.98 & $1.50 \times 10^{-3}$ & 0.50 \\
\hline 城市 & 荆门 & 随州 & 仙桃 & 宜昌 & 天门 & 恩施州 & 十堰 & 潜江 \\
\hline$c_{1}$ & 42.77 & 50.98 & 37.93 & 26.91 & 47.99 & 21.89 & 35.94 & 60.01 \\
\hline$q_{1}$ & $2.75 \times 10^{-6}$ & $1.04 \times 10^{-9}$ & $2.11 \times 10^{-9}$ & $7.07 \times 10^{-8}$ & $1.58 \times 10^{-6}$ & $2.77 \times 10^{-6}$ & $1.76 \times 10^{-6}$ & $9.49 \times 10^{-7}$ \\
\hline$c_{2}$ & 1.01 & 8.00 & 70.99 & 1.00 & 47.99 & 1.00 & 1.00 & 50.02 \\
\hline$q_{2}$ & $1.00 \times 10^{-5}$ & $1.27 \times 10^{-6}$ & $1.88 \times 10^{-9}$ & $1.00 \times 10^{-5}$ & $6.78 \times 10^{-6}$ & $1.02 \times 10^{-46}$ & $1.00 \times 10^{-5}$ & $1.52 \times 10^{-9}$ \\
\hline$c_{3}$ & 1.01 & 0.10 & 24.38 & 0.03 & 47.98 & 0.01 & 0.01 & 2.00 \\
\hline$q_{3}$ & $9.46 \times 10^{-4}$ & $9.99 \times 10^{-4}$ & $1.00 \times 10^{-3}$ & $9.86 \times 10^{-4}$ & $1.00 \times 10^{-3}$ & $1.05 \times 10^{-3}$ & $9.68 \times 10^{-4}$ & $1.52 \times 10^{-9}$ \\
\hline$R_{c 1}$ & 3.61 & 2.79 & 1.46 & 2.77 & 1.90 & 2.17 & 3.01 & 1.42 \\
\hline$R_{c 2}$ & 0.03 & 0.46 & 2.90 & 0.10 & 1.67 & 0.09 & 0.06 & 1.17 \\
\hline$R_{c 3}$ & 0.04 & $5.00 \times 10^{-3}$ & 0.99 & $2.80 \times 10^{-3}$ & 0.83 & $7.54 \times 10^{-4}$ & $6.33 \times 10^{-4}$ & 0.05 \\
\hline
\end{tabular}

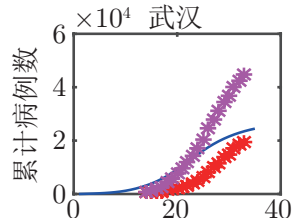

威宁
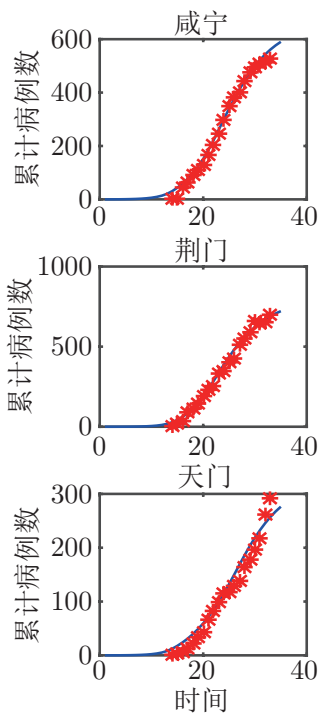

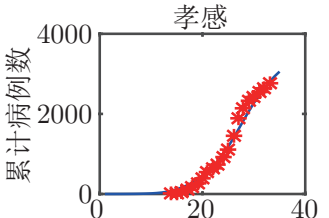

鄂州
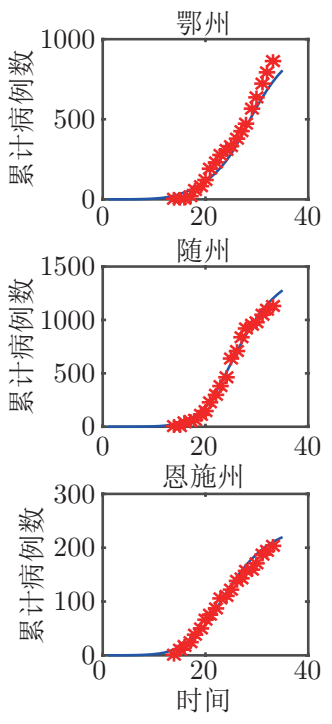

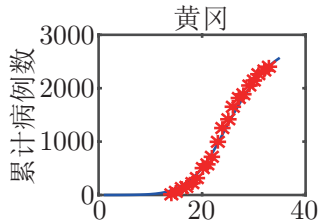

襄阳
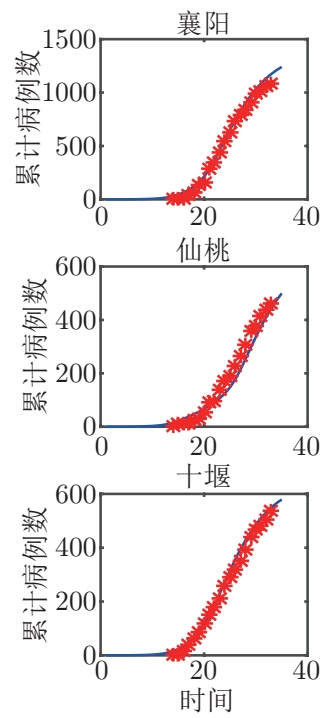

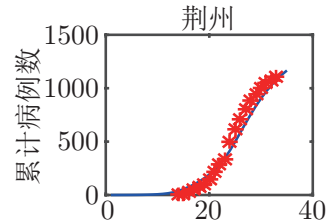

黄石
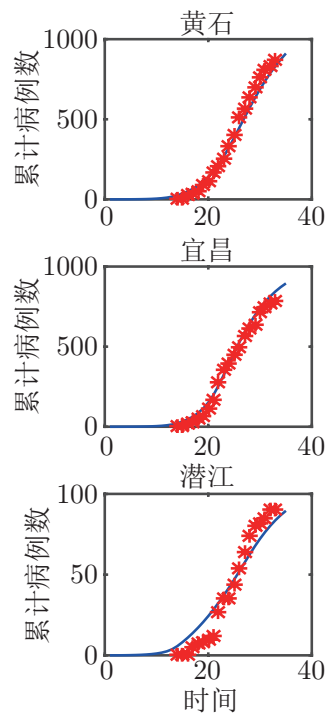

图 3 (网络版彩图) 模型拟合的结果. 蓝线是模拟结果, 红星号是各个城市的累计数据, 紫色星号是全国累计数据 


\section{3 主要结果}

在 COVID-19 疫情初期, 由于研制核酸检测试剂盒、检出和确诊等都需要时间, 报告病例数明显 低于实际的病例数, 而且早期全国其他地区的病例主要来自武汉, 因此, 从其他城市的病例数来估计武 汉的实际病例数至关重要, 根据网络模型拟合, 我们得到 1 月 23 日武汉累计病例数的估计值为 2214 例, 几乎是我国 23 日累计报告病例数 (830 例) 的 3 倍, 这说明在武汉封城时疫情就比较严重.

根据估计的参数值, 我们得到了湖北省 16 个城市早期 (第一阶段) 的控制再生数 (见表 3), 从表 中可知武汉早期的控制再生数为 3.66, 其他城市的控制再生数均小于武汉. 这里估计武汉的再生数较 小 (参见文献 [4]), 一方面由于有一部分病例已经流入全国的其他城市, 另一方面核酸检测试剂盒并没 有检出很多病例 (参见文献 [13]). 早期控制再生数居前 5 位的城市 (武汉除外) 是荆门、十堰、随州、 襄阳和宜昌. 为了研究不断增强的防控措施对新发感染的影响, 我们还估计了第二、三阶段的控制再 生数. 结果表明多数城市的控制再生数明显下降, 特别是目前各个城市的控制再生数均小于 1 ; 表明目 前新发感染非常低, 疫情在各地得到有效控制. 需要说明的是第三阶段新增病例数已有明显的下降趋 势, 因此, 个别城市的控制再生数估计值非常小.

为了研究复工对湖北省境内各个城市疫情的影响, 我们基于湖北省境内各个城市 2019 年同期的 流动网络结构和流动量模拟复杂网络模型 (2.2), 而且分别考虑了不同的复工时间以及控制强度的影 响 (分别取 $c_{1}, q_{1}$ 和 $c_{2}, q_{2}$ ). 2019 年 2 月 8 日至 3 月 1 日武汉人口迁入时间分布如图 1(b) 所示, 迁 入人口来源地分布假设与 2020 年 1 月 10 日至 1 月 23 日武汉迁出人口目的地分布相同 (如图 2 所 示). 模拟时只考虑各城市人口迁入武汉, 不考虑迁出, 假设迁入武汉总人口等于年前迁出武汉总人口. 假设复工时间为 2 月 17 日, 人口流动则从 2 月 14 日开始持续至 3 月 3 日 (对应 2019 年 2 月 8 日 至 3 月 1 日的流动趋势). 图 4-6 分别给出了 2 月 17 日、 2 月 24 日和 3 月 2 日的复工对各个城市疫 情的影响, 从图 4 和 5 可以看出, 除了黄冈和天门之外, 复工会使其他城市的病例数有明显增加, 对疫
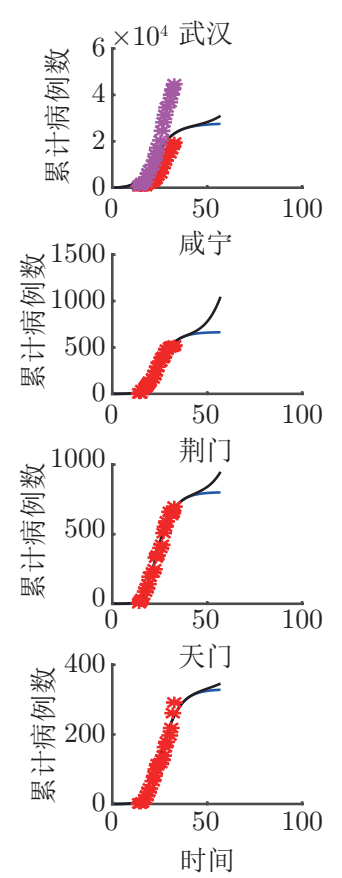
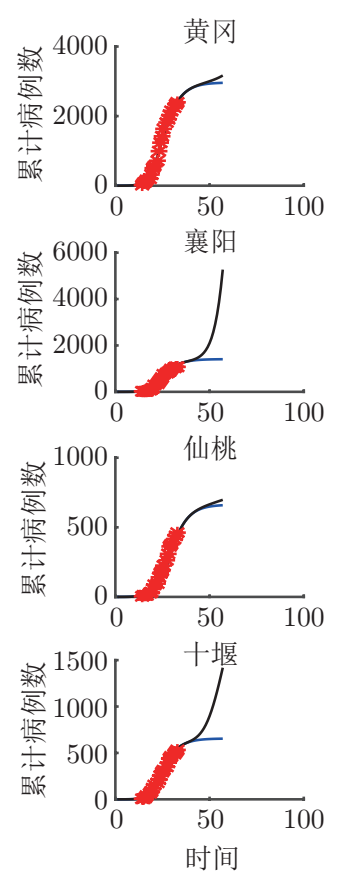
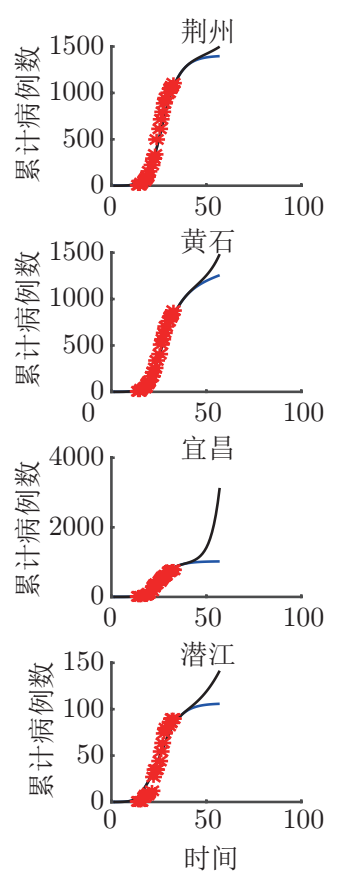

图 4 (网络版彩图) 复工对各个城市疫情的影响. 蓝线表示没有复工的模拟结果, 黑线表示在 2 月 17 日复工的模拟 结果, 红星号是各个城市的累计数据, 紫色星号是全国累计数据, 参数选取早期 $\left(c_{1}\right.$ 和 $\left.q_{1}\right)$ 的参数值 

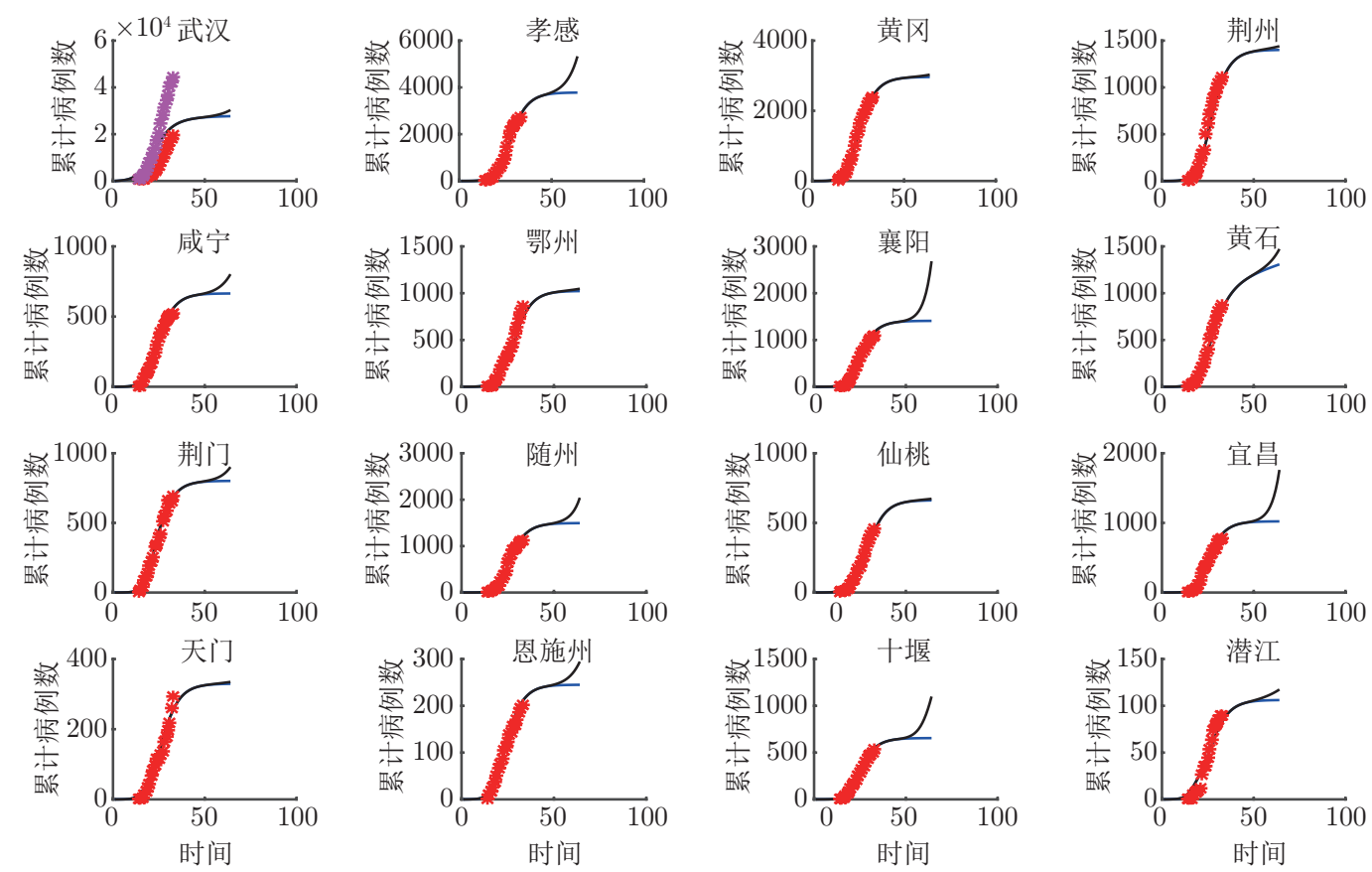

图 5 (网络版彩图) 复工对各个城市疫情的影响. 蓝线表示没有复工的模拟结果, 黑线表示在 2 月 24 日复工的模拟 结果, 红星号是各个城市的累计数据, 紫色星号是全国累计数据, 参数选取早期 $\left(c_{1}\right.$ 和 $\left.q_{1}\right)$ 的参数值
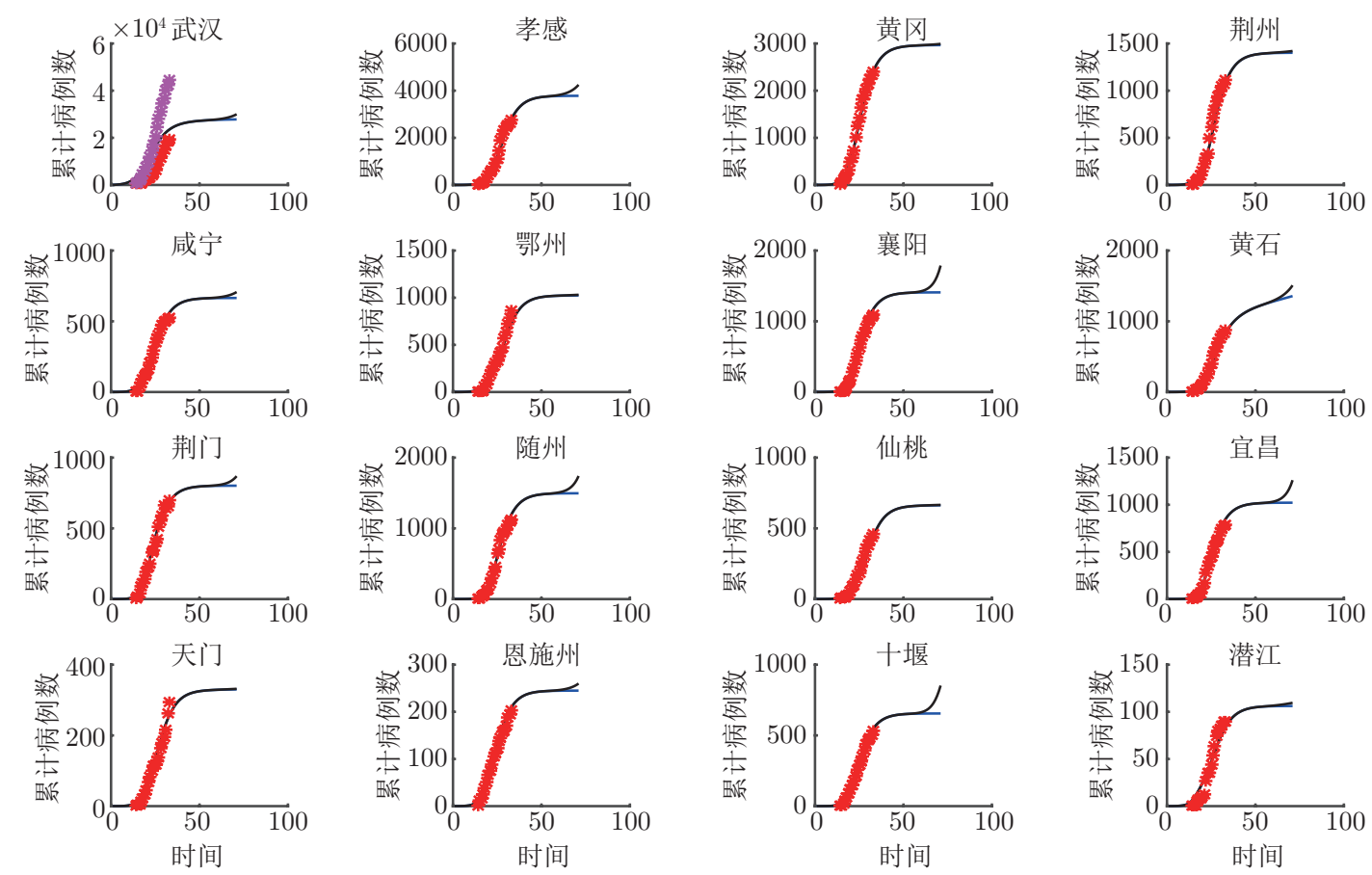

图 6 (网络版彩图) 复工对各个城市疫情的影响. 蓝线表示没有复工的模拟结果, 黑线表示在 3 月 2 日复工的模拟 结果, 红星号是各个城市的累计数据, 紫色星号是全国累计数据, 参数选取早期 ( $c_{1}$ 和 $\left.q_{1}\right)$ 的参数值

情不利; 从图 6 可以看出, 3 月 2 日复工如果控制措施不够严格, 复工约 10 天后累计病例数会有较明 显的增加, 多数城市的疫情会在 20 天内再次暴发. 但是若复工时再加强防控措施 (基于中期的参数), 

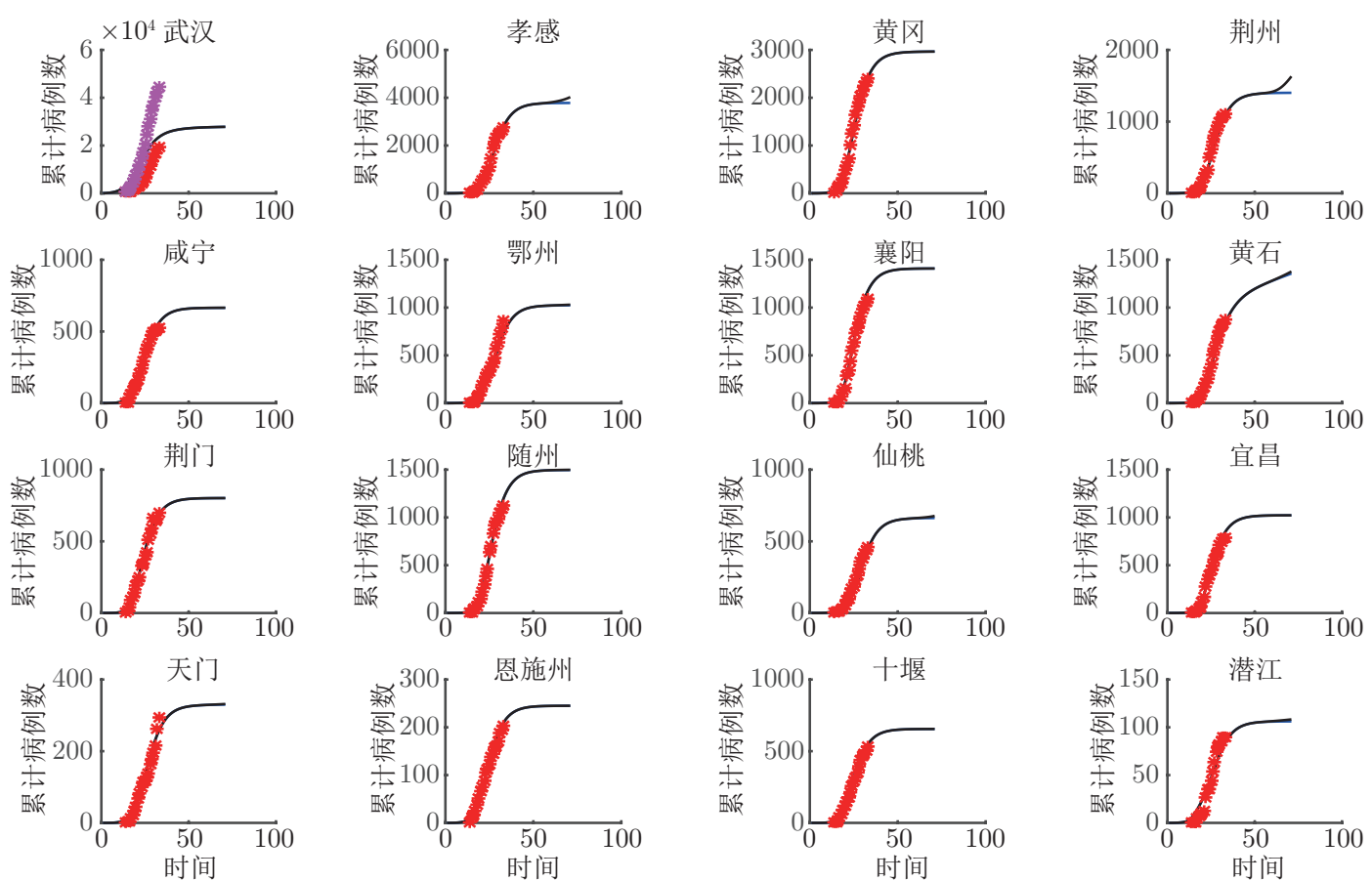

图 7 (网络版彩图) 复工对各个城市疫情的影响. 蓝线表示没有复工的模拟结果, 黑线表示在 3 月 2 日复工的模拟 结果, 红星号是各个城市的累计数据, 紫色星号是全国累计数据, 参数选取中期 $\left(c_{2}\right.$ 和 $\left.q_{2}\right)$ 的参数值

从图 7 可以看出, 3 月 2 日的复工在较长的时间内除了个别城市如荆州、孝感、黄石病例数略有上浮 外, 不会引起其他各个城市疫情的二次暴发.

\section{4 总结和何时复工建议}

春节后的 COVID-19 疫情在全国各地围堵缓疫防控措施下, 疫情的蔓延趋势得到有效控制. 但随 之而来的严峻问题是: 在保证因为人员流动导致二次暴发的风险较低的情况下, 返乡人员特别是武汉 城区流入到周边疫情较严重区域的人员何时能够返回原地复工? 因此, 本文基于国家和湖北省卫健委 报告的相关疫情数据, 根据百度迁徙网站上 2019 年及 2020 年的人口流动趋势与分布大数据, 得到了 武汉春节前人员流出和春节后人员流入的趋势. 然后构建武汉及周边 15 个疫情严重城市的 COVID-19 传播复杂网络模型.

由于武汉周边疫情严重城市自 1 月 26 日开始才相继加强措施并报告完整的疫情数据, 因此, 为 了评估不断加强的防控措施的有效性, 我们分阶段给出了 16 个城市的控制再生数. 结论显示, 第一个 阶段所有地区控制再生数均大于 1 , 这意味着传播风险高; 第二个阶段有 9 个城市小于 1 , 控制初步见 效; 第三个阶段的控制再生数均小于 1 (见表 3), 则新发感染率全面降低. 然后分别在时间节点 2 月 17 日、2 月 24 日和 3 月 2 日变化网络节点中的关键参数 (接触数 $c$ 和隔离率 $q$ 变为 1 月 23 日封城 之前的估计值), 并依据百度迁徙网站给出的春节返程流入武汉的趋势增加流入率, 评估复工导致二次 暴发的风险. 主要结论显示湖北地区的复工不能早于 3 月 2 日, 否则疫情可能二次暴发. 若 3 月 2 日 复工并有较强的防控措施, 湖北各个城市的疫情在较长时间内将不会二次暴发.

致谢 特别感谢审稿人提出的宝贵意见, 感谢研究生杨洁和赵吴琼在研究期间收集数据所付出的努力. 
1 World Health Organization (WHO). Coronavirus. Https://www.who.int/health-topics/coronavirus, 2020

2 World Health Organization (WHO). Situation report. Https://www.who.int/docs/default-source/coronaviruse/ situation-reports/20200123-sitrep-3-2019-ncov.pdf, 2020

3 严阅, 陈瑜, 刘可伋, 等. 基于一类时滞动力学系统对新型冠状病毒肺炎疫情的建模和预测. 中国科学: 数学, 2020, 50: $385-392$

4 Tang B, Wang X, Li Q, et al. Estimation of the transmission risk of the 2019-nCoV and its implication for public health interventions. J Comput Math, 2020, 9: 462

5 Tang B, Bragazzi N L, Li Q, et al. An updated estimation of the risk of transmission of the novel coronavirus (2019-nCoV). Infect Dis Model, 2020, 5: 248-255

6 Xiao Y, Tang S, Wu J. Media impact switching surface during an infectious disease outbreak. Sci Rep, 2015, 5: 7838

7 湖北省卫生健康委员会. 2020 年 2 月 12 日湖北省新冠肺炎疫情情况. Http://wjw.hubei.gov.cn/fbjd/dtyw/202002/ t20200213_2025581.shtml, 2020

8 中华人民共和国国家卫生健康委员会. 疫情通报. Http://www.nhc.gov.cn/xcs/xxgzbd/gzbd_index.shtml, 2020

9 World Health Organization (WHO). Statement on the meeting of the International Health Regulations (2005) Emergency Committee regarding the outbreak of novel coronavirus (2019-nCoV). Https://www. who.int/news-room/detail/23-01-2020-statement-on-the-meeting-of-the-international-health-regulations-(2005) -emergency-committee-regarding-the-outbreak-of-novel-coronavirus-(2019-ncov), 2020

10 Tang B, Fan X, Tang S, et al. The evolution of quarantined and suspected cases determines the final trend of the 2019-nCoV epidemics based on multi-source data analyses. Https://ssrn.com/abstract=3537099, 2020

11 Eisenberg M C, Shuai Z, Tien J H, et al. A cholera model in a patchy environment with water and human movement. Math Biosci, 2013, 246: 105-112

12 马之恩, 周义仓. 传染病动力学的数学建模与研究. 北京: 科学出版社, 2004

13 湖北省卫生健康委员会. 武汉: 单日样本检测能力从 200 份提升至 2000 份. Http://wjw.hubei.gov.cn/bmdt/ztzl/ fkxxgzbdgrfyyq/fkdt/202001/t20200129_2016053.shtml, 2020

\section{When will be the resumption of work in Wuhan and its surrounding areas during COVID-19 epidemic? A data-driven network modeling analysis}

\section{Xia Wang, Sanyi Tang, Yong Chen, Xiaomei Feng, Yanni Xiao \& Zongben Xu}

Abstract Based on the report of epidemic data sets for Hubei province and mainland China, the big data from Baidu population migration trends and distributions, we formulated the COVID-19 transmission model on complex networks for Wuhan city and the 15 surrounding cities with severe epidemics, and analyzed the possible times for resumption of work in Wuhan and its surrounding areas and the impact of resumption of work on the risk of a secondary outbreak. Firstly, we estimated the actual cumulative number of cases in Wuhan on January 23rd on the basis of the cumulative number of reported cases in other 15 cities, and obtained the control reproduction numbers for the 16 major cities in Hubei province in different periods. Our research results revealed that the early transmission risk in these areas is high and the current transmission risk is low (due to the control reproduction numbers being less than 1). By simulating the whole network model on the flow network structure and flow volume in the same period of last year, we investigated the impact of the resumption of work on February 17, February 24 and March 2 on disease infection for each city. Main conclusion showed that with strong prevention measures and self-protection the second outbreak will not be caused during a period by the resumption of work on March 2, 2020.

Keywords novel coronavirus pneumonia, network modelling, controlling reproduction number, epidemic prediction

MSC(2010) 35A34, 65N21

doi: $10.1360 / S S M-2020-0037$ 